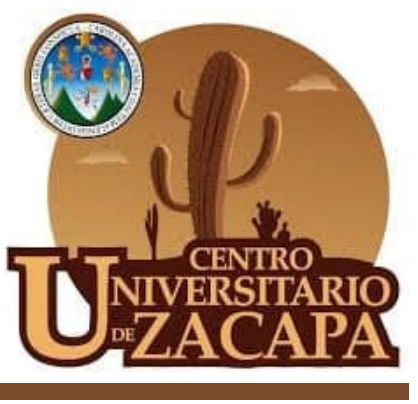

Recibido: $15 / 11 / 2020$

Ensayo científico

Publicado: 16/01/2021

\title{
Enfoque de la valoración neurogeriátrica inte- gral en el envejecimiento saludable
}

\section{Comprehensive Neurogeriatric Assessment Approach to Heal- thy Aging}

Jorge Eduardo Bran Girón

Universidad de San Carlos de Guatemala

dr.jorgeduardojr@hotmail.com

https://orcid.org/0000-0001-7652-9240

\section{Referencia del ensayo}

Bran Girón, J. E. (2021). Enfoque de la valoración neurogeriátrica integral en el envejecimiento saludable. Revista Académica CUNZAC, 4(1), 75-80. DOI: https://doi.org/10.46780/cunzac.v4i1.35

\section{Resumen}

El envejecer de manera exitosa esta relacionado con llevar una vida saludable y modificar estilos de vida que puedan reflejar enfermedades crónicas degenerativas; la población adulta mayor que se define en Guatemala como persona de sesenta años. Este grupo poblacional necesita de una atención para la promover el envejecimiento saludable con el fin de aplicar modelos neurocientíficos orientados a la detección temprana de enfermedades crónicas y al tratamiento adecuado integrado por profesionales de las neurociencias de enfoque multidisciplinario que conforman planes de prevención y ejecución para el éxito del envejecimiento que es dar calidad de vida. La atención oportuna para el envejecimiento saludable es valorada mediante el vínculo de la capacidad visual, auditiva, cognitiva, locomotriz, psicológica y vital relacionada con estrategias de calidad de atención que se encargan de brindar el bienestar físico, mental y emocional de las personas mayores enfocándolos a un mundo más inclusivo hacia las diferentes disciplinas de las neurociencias. El enfoque de la valoración geriátrica mediante un equipo multidisciplinario es la visión estratégica de éxito del envejecimiento saludable desde el punto de vista de las neurociencias.

Palabras clave: neurociencias, geriatría, gerontología, sarcopenia, dependencia, fragilidad, demencia, vida

\begin{abstract}
Aging successfully is related to leading a healthy life and modifying lifestyles that may reflect chronic degenerative diseases; the older adult population defined in Guatemala as a person over sixty years of age. This population group needs attention to promote healthy aging in order to apply neuroscientific models aimed at the early detection of chronic diseases and appropriate treatment integrated by professionals from the neurosciences with a multidisciplinary approach that make up prevention and implementation plans for the success of aging which is to give quality of life. Timely care for healthy aging is valued by linking visual, auditory, cognitive, locomotor, psychological and vital capacity related to quality of care strategies that are responsible for providing the physical, mental and emotional well-being of the elderly by focusing on them to a more inclusive world towards the different disciplines of neuroscience. The approach to geriatric assessment by a multidisciplinary team is the strategic vision for the success of healthy aging.
\end{abstract}

Keywords: neurosciences, geriatrics, gerontology, sarcopenia, dependence, frailty, dementia, life 


\section{Introducción}

El término neurociencias es relacionado con múltiples disciplinas que se encargan del estudio del sistema nervioso como un todo y es así como se relaciona con el estudio del comportamiento en diferentes áreas de la neurocognición. Por consiguiente, la necesidad del estudio de se debe al engranaje de procesos cognitivos fisiológicos y patológicos que existen a nivel mundial.

El informe mundial sobre el envejecimiento y la salud en el 2015 estableció metas para el fomento del envejecimiento activo saludable que se atribuye a la independencia tanto intrínseca, entorno y relaciones intergeneracionales por lo tanto en el 2017 realizan recomendaciones mediante medicina basada en evidencia por tanto es justificable relacionarlo con las neurociencias por la necesidad de una atención integrada a las personas mayores que en Guatemala son definidas como personas de sesenta años y más (OPS, 2020).

El objetivo establecer la aplicación de la valoración geriátrica integral neurocientífica por parte de profesionales para una atención oportuna y de calidad del envejecimiento saludable.

\section{Contenido}

El término envejecimiento exitoso se refiere a las personas adultas mayores que no padecen de enfermedad cronicodegenerativas y en ausencia de síndromes geriátricos que promuevan la dependencia.
Es así como la organización mundial de la salud (OMS) indica que a partir del año 2021 al 2030 será la década del envejecimiento saludable que se relaciona con el cumplimiento de los Objetivos del Desarrollo Sostenible (ODS) siendo estos metas claves para el desarrollo en tres dimensiones social, ambiental y económico (Organización Mundial de la Salud, 2020).

En efecto las neurociencias influyen en la formación de profesionales que se encargan del desarrollo de proyectos encaminados a mejorar los procesos neurocognitivos desde el punto de vista social, económico, cultural y en el sector salud. Siendo capaces de identificar características en ciertos grupos de población a los cuales dirijan sus servicios.

Las neurociencias en geriatría son sumamente importantes siendo vitales en la promoción del envejecimiento saludable y en la reducción en este grupo vulnerable de la aparición de síndromes geriátricos relacionados con la aparición de envejecimiento patológico (Ibid). 
Tabla No.1 Relación de los grandes síndromes geriátricos y el envejecimiento patológico

\begin{tabular}{ll}
\hline Envejecimiento Patológico \\
\hline Síndrome geriátrico & Definición \\
\hline Sarcopenia & Pérdida de masa muscular \\
\hline Fragilidad & Decremento de las reservas fisiológicas que \\
& comprometen la integridad y aceleran el agotamiento \\
& físico del adulto mayor. \\
\hline Dependencia & Disminución de la capacidad de realizar actividades de \\
& la vida diaria. \\
\hline Polifarmacia & Uso simultaneo de grandes cantidades de fármacos. \\
\hline Inmovilidad & Deterioro funcional e incapacidad para trasladarse o \\
& movilizarse de manera autónoma. \\
\hline Deterioro cognitivo & Síndrome caracterizado por la pérdida de las \\
& funciones cognitivas \\
& neurodegenerativa primaria o secundaria. \\
\hline Institucionalización & Pérdida involuntaria de retención de orina y heces. \\
\hline Depresión & Síndrome caracterizado por afección del estado físico \\
& y mental vinculado con dificultades de la esfera social \\
& y su entorno. \\
\hline & Ingreso de un adulto mayor a una residencia geriátrica \\
\hline
\end{tabular}

Fuente: elaboración propia con base a Manual de Residentes de Geriatría de la Asociación Española de Geriatría y Gerontología AEGG.

Los adultos mayores han sido grupo vulnerable por años, es más el envejecimiento se percibía como una enfermedad; sin embargo, la evidencia científica nos indica que existen factores modificables que se implican en los cambios epigenéticos prácticamente es ofrecer calidad de vida. El término dar calidad de vida se refiere a que estos cambios deben de promoverse mediante hábitos saludables que se deben de dar desde la niñez, adolescencia y en la etapa adulto joven para poder disminuir el aparecimiento de síndromes geriátricos devastadores en etapa del envejecimiento y poder promover un envejecimiento saludable (Sociedad Española de Geriatría y Gerontología, 2017). El Manual ICOPE se caracteriza por la valoración integral de la población adulta mayor y lo realiza mediante 6 capacidades siendo este una estrategia y cumplimiento del envejecimiento y salud en lograr los objetivos del desarrollo sostenible siendo pilares tres factores importantes a) dar el derecho a las personas mayores de una salud de calidad, b) Promover la igualdad para acceder a determinantes del envejecimiento saludable como tal en sector económico, social e infraestructura de centros de atención c) Atención igualitaria sin discriminación por edad o género.

Las seis capacidades anteriormente mencionadas es la valoración integrada de la capacidad vital, auditiva, visual, cognitiva, psicológica y locomotriz, estas se logran mediante la formación de equipos multidisciplinarios en el sector geriátrico. 
Cada vez estamos convencidos que la necesidad de las evaluaciones constantes en la población en general es realmente importante esto con el objeto de la promoción primaria en Salud y programas que promuevan el envejecimiento saludable. Los puntos claves para el logro en neurociencias es primordial enfatizar en 9 aspectos realmente importantes que van dirigidos a mejorar la salud y dar calidad de vida a las personas mayores.

Figura No.1 Modelo de atención integral en envejecimiento saludable

Modelo de atención integral en envejecimiento saludable
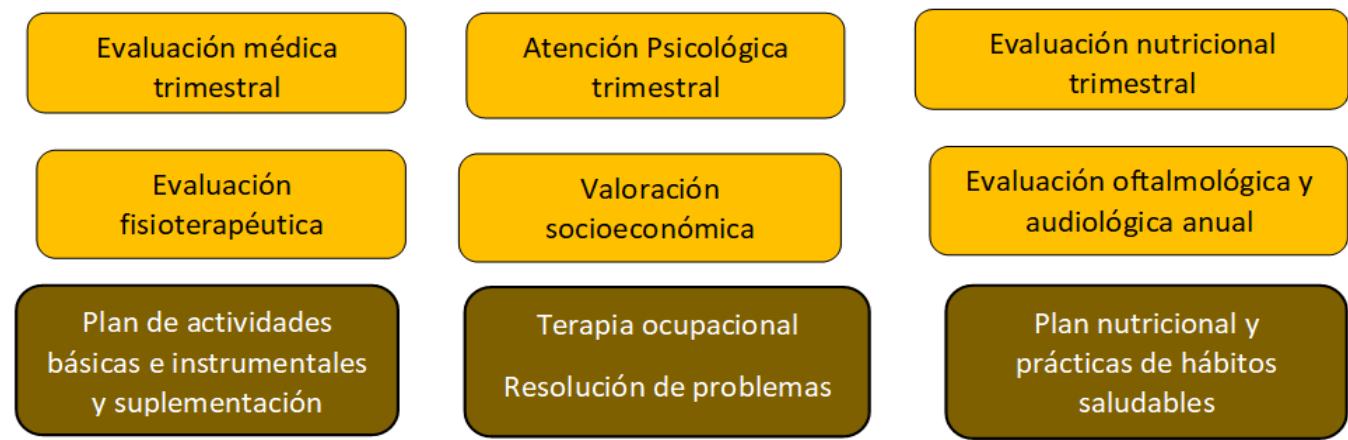

Evaluación oftalmológica y audiológica anual

Plan nutricional y

prácticas de hábitos

saludables

Fuente: elaboración propia, con base al Manual de ICOPEL Atención Integrada para las personas mayores.

El enfoque neurocientífico va enfocado en la creación de equipos multidisciplinarios con relación a la promoción del envejecimiento saludable y el evitar la pérdida de las funciones cognitivas es por ello que se desglosara cada uno de los nueve elementos siendo estos últimos tres un enfoque integrado.

La evaluación médica trimestral es necesaria hoy en día se conocen los programas de brigadas ambulatorios o bien la atención centrada en ciertos programas promoviendo el envejecimiento saludable en el cual no solamente la exploración física juega un papel importante sino también el tamizaje bioquímico y hematológico asimismo como la suplementación de multivitaminas, ácidos omega 3,6 y 9 así como el aporte de vitamina $D$ como es conocido y es científicamente comprobado la suplementación anteriormente mencionada promueve a mejorar las funciones neurocognitivas de los adultos mayores asimismo disminuyendo los riesgos metabólicos entre ellos la hipertensión arterial sistémica, diabetes mellitus, arteriosclerosis, demencia primarias y secundarias, asimismo el aparecimiento de enfermedades autoinmunes y hematooncológicas. Se realiza hincapié en la valoración conjunta con fisioterapia para la medición de amplitudes articulares, el promover un plan de ejercicios para la reducción de riesgos metabólicos pero orientado a la ganancia de masa muscular y evitar la dependencia asimismo como los estados de fragilidad, el envejecimiento saludable se evidencia no solo en la atención personalizada sino también centros de respiro, residencias geriátricas que conjuntamente con la valoración de estado funcional por medio de los cuidadores y familiares desarrollan habilidades para promover el estado de independencia como tal. 
Posteriormente un elemento importante es la evaluación psicológica porque por medio de esta valoración se conoce el entorno social, familiar y económico asimismo el vínculo que se relaciona con problemas de índole somático y psicosociales, la terapia psicogerontológica ha tenido un auge cada vez mayor en los últimos años mejorando el bienestar mental del adulto mayor, así como la resiliencia a la adaptación de los cambios fisiológicos del mismo. La valoración sociogerontológica va encaminada en determinar el entorno del adulto mayor si bien es sabido el conocer las barreras arquitectónicas, el estado de la vivienda, el contar con los servicios públicos se vuelve tan necesarios para evitar accidentes dentro de casa y es más asociar a adultos mayores en el beneficio de ciertos programas sociales, médicos, nutricionales y económicos que pueden recibir para la mejora de su vida. El factor nutricional en adultos mayores es sumamente importante la detección de enfermedades como la sarcopenia, obesidad sarcopenia y el aparecimiento de enfermedades cronicodegenerativas es vital, sin em- bargo la valoración nutricional mediante las pruebas antropométricas es necesario así como el seguimiento trimestral para detección temprana de los síndromes anteriormente mencionados.

La importancia de la evaluación oftalmológica y auditiva debe ser inclusiva a todo nivel por la perdida de la capacidad visual y la capacidad auditiva estas capacidades se pierden conforme la edad avanza sin embargo existen tratamientos oportunos y el uso de aparatos auditivos y visuales que nos apoyan a dar una calidad de vida e inclusión a este grupo vulnerable. Los últimos tres elementos conciernen a un plan integrado en plan de actividades de vida diaria, suplementación médica, resolución de problemas del entorno, terapia ocupacional finalizando con el apoyo integrado nutricional y practicas saludables que conciernen posteriormente a la valoración geriátrica integrada que se relaciona con diferentes escalas e índices que se presentan a continuación.

Tabla No. 2 Metodología para la evaluación geriátrica de calidad mediante la capacidad

\begin{tabular}{ll}
\hline Capacidad & Métodos de valoración geriátrica \\
\hline Capacidad Cognitiva & $\begin{array}{l}\text { Minimental Test de Folstein Modificado } \\
\text { Test de Pfifer } \\
\text { Valoración de las esferas mentales }\end{array}$ \\
\hline Capacidad Psicológica & DMS V y test orientados a depresión, \\
& ansiedad (Síndrome del nido vacío) \\
\hline Capacidad locomotríz & Indice de Barthel, escala de Lawton \\
\hline Capacidad Vital & Brody, cruz roja e índice de Katz \\
\hline Capacidad Auditiva & Mini Nutritional Assesment y medición \\
\hline Capacidad Visual & antropometríca ISAK \\
\hline
\end{tabular}

Fuente: elaboración propia con base al Manual de ICOPEL Atención Integrada para las personas mayores 
Es evidente que la funcionalidad es parte de la neurociencia y conlleva a una estructura de atención integrada la valoración geriátrica siempre será la herramienta eficaz para la evaluación de los adultos mayores con el fin de establecer no solamente planes curativos sino preventivos incluidos el envejecimiento exitoso.

\section{Conclusión}

Las neurociencias son disciplinas evolutivas que se encargan de crear modelos de atención inclusivos, en el caso de la población geriátrica siempre se desarrollan mediante un fin que es brindar calidad de vida.

El envejecimiento saludable debe de aplicarse mediante un programa preventivo que incluya equipo multidisciplinario con enfoque neurocientífico y neurorrehabilitación para poder cumplir con las metas claves que otorga la atención geriátrica especializada.

La aparición de los grandes síndromes geriátricos se dará siempre y cuando no se realicen las modificaciones del estilo de vida que conlleve a enfermedades crónico degenerativas.

\section{Referencias}

OPS. (2020). Manual Guía sobre la evaluación y esquemas de atención centrados en la persona en atención primaria en salud. ICOPE, 96. Recuperado el 31 de marzo de 2021, de file:///C:/Users/HP/Downloads/OPSFPLHL200004A_spa.pdf

Organización Mundial de la Salud. (2020). Organización Mundial de la Salud. Obteni- do de https://www.who.int/es/initiatives/ ageing/decade-of-healthy-ageing

Sociedad Española de Geriatría y Gerontología. (2017). Sociedad Española de Geriatría y Gerontología. Recuperado el 31 de marzo de 2021, de https://www. segg.es/institucional/2017/05/29/el-envejecimiento-como-oportunidad-y-no-como-problemas

\section{Sobre el autor}

Es maestro en Neurociencias Médicas y Geriatría y Gerontología, se desempeña como docente en la Universidad de San Carlos de Guatemala.

\section{Copyright (c) (2021) Jorge Eduardo Bran Girón}

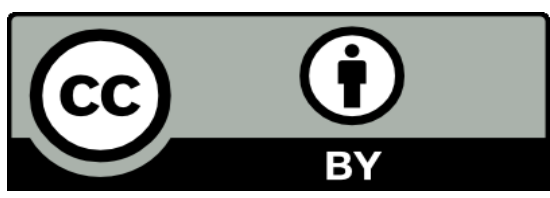

Este texto está protegido por una licencia Creative Commons 4.0.

Es libre para compartir, copiar y redistribuir el material en cualquier medio o formato y adaptar el documento, remezclar, transformar y crear a partir del material para cualquier propósito, incluso comercialmente, siempre que cumpla la condición de atribución: debe reconocer el crédito de una obra de manera adecuada, proporcionar un enlace a la licencia, e indicar si se han realizado cambios. Puede hacerlo en cualquier forma razonable, pero no de forma tal que sugiera que tiene el apoyo del licenciante o lo recibe por el uso que hace. 